NeLSON ElIAS ANDRADE JUNIOR ${ }^{1}$

MARIA LÚCIA ELIAS PIRES ${ }^{2}$

Luiz Claudio Santos Thuler ${ }^{3}$

Artigo original

Palavras-chave

Depressão/etiologia

Ansiedade/etiologia

Hipotireoidismo/complicações

Keywords

Depression/etiology

Anxiety/etiology

Hypothyroidism/complications

\section{Sintomas depressivos e ansiosos em mulheres com hipotireoidismo}

\author{
Depression and anxiety symptoms in bypotbyroid women
}

\section{Resumo}

OBJETIVO: avaliar a associação entre hipotireoidismo e a ocorrência de sintomas depressivos e ansiosos. MÉTODOS: foi realizado um estudo do tipo caso-controle, no período de julho de 2006 a março de 2008, no qual foram incluídas 100 mulheres (50 pacientes com hipotireoidismo primário e 50 controles eutireoidianas) com idade entre 18 e 65 anos. Foram avaliados idade, raça/cor da pele, estado civil, nível educacional, consumo de álcool, situação de trabalho, índice de massa corpórea e estado menopausal. Foram realizadas dosagens de TSH e utilizadas as escalas de ansiedade e de depressão de Beck em todos os casos e controles. O programa utilizado para a análise estatística foi o SPSS, versão 14. O nível de significância adotado foi p<0,05. RESULTADOS: não foram verificadas diferenças significativas entre pacientes com hipotireoidismo primário e controles no que se refere às variáveis demográficas e epidemiológicas. A presença concomitante de ansiedade e depressão foi cinco vezes maior entre os casos do que entre os controles (20 versus 4\%; $p=0,01$ ). A ocorrência de sintomas ansiosos foi cerca de três vezes maior entre os casos (40\%) em relação aos controles (14\%) (p=0,003), enquanto a prevalência de sintomas depressivos mostrou-se $75 \%$ superior entre casos (28\%) quando comparada aos controles (16\%) (p=0,15). Neste estudo não foi observada associação entre os níveis de TSH e a prevalência de sintomas de ansiedade e depressão. CONCLUSÕES: este estudo caso-controle apontou uma maior probabilidade de pacientes com hipotireoidismo apresentarem sintomas ansiosos e depressivos em comparação a controles eutireoidianas. Devido às altas prevalências de hipotireoidismo e depressão observadas na prática clínica, a presença de sintomas depressivos deve ser investigada em pacientes com disfunção tireoidiana e pacientes deprimidos devem ser testados com dosagem do TSH.

\section{Abstract}

PURPOSE: to study the association between hypothyroidism and depression and anxiety symptoms. METHODS: a case-control study was carried out from July 2006 to March 2008 on 100 patients 150 patients with primary hypothyroidism and 50 euthyroid controls) aged 18 to 65 years. Age, race/skin color, marital status, education level, alcohol use, working status, body mass index and menopausal status were evaluated. TSH levels were determined and the Beck Depression and Beck Anxiety Scales were applied to all cases and controls. Statistical analysis was performed using the SPSS software version 14.0. The level of significance was set at $p<0.05$. RESULTS: there was no demographic or epidemiologic difference between groups. The concomitant presence of anxiety and depression was five times greater among cases than among controls $(20.0$ versus $4.0 \%, p=0.01)$. Anxiety symptoms were approximately three times more frequent among cases $(40.0 \%)$ than among controls (14.0\%) ( $\mathrm{p}=0.003)$, while the prevalence of depressive symptoms was $75 \%$ higher among cases (28.0\%) than among controls (16.0\%), but this did not reach statistical significance ( $p=0.15)$. We found no association between TSH levels and the prevalence of anxiety or depression symptoms. CONCLUSIONS: this case-control study showed a greater probability for hypothyroid patients to develop anxiety and depression symptoms when compared to euthyroid controls. Due to the high prevalence of hypothyroidism and depression observed in clinical practice, depressive symptoms must be considered in patients with thyroid dysfunction and depressed patients should be tested for TSH.
Correspondência:

Luiz Claudio Santos Thuler

Pós-Graduaç̃o em Neurologia da Universidade Federal do Estado do

Rio de Janeiro (UNIRIO)

Rua Mariz e Barros, 775 - Tijuca

(EP 20270-004 - Rio de Janeiro (RJ), Brasi

E-mail: Ithuler@gmail.com

Conflito de interesse: não há
Hospital Universitário Gaffrée e Guinle - Programa de Pós-Graduação em Neurologia Stricto Sensu da Universidade Federal do Estado do Rio de Janeiro - UNIRIO - Rio de Janeiro (RJ), Brasil.

Mestre em Neurologia pela Universidade Federal do Estado do Rio de Janeiro - UNIRIO; Médico Clínico Geral do Corpo de Saúde da Marinha - Rio de Janeiro (RI), Brasil.

2 Professora Associada e Coordenadora do Curso de Pós-Graduação Latu Sensu em Endocrinologia da Universidade Federal do Estado do Rio de Janeiro - UNIRIO - Rio de Janeiro (RJ), Brasil.

3 Professor Adjunto da Universidade Federal do Estado do Rio de Janeiro - UNIRIO; Coordenador de Educação e Professor da PósGraduação em Oncologia do Instituto Nacional de Câncer - INCA - Rio de Janeiro (RJ), Brasil. 


\section{Introdução}

O hipotireoidismo tem sido reconhecido como importante causa de morbidade no sexo feminino, atingindo 4 a $10 \%$ de todas as mulheres ${ }^{1}$. No Brasil, estudo envolvendo 1.298 mulheres de meia idade vivendo em área metropolitana mostrou que 12,3\% apresentavam a doença ${ }^{2}$. Em outros estudos nacionais, a prevalência tem variado entre $5^{3}$ e $17,6 \%{ }^{4}$ e, entre mulheres climatéricas, foi observada uma taxa de $16,1 \%$ em mulheres na pós-menopausa ${ }^{5}$. No que diz respeito às gestantes, estima-se que 5 a $15 \%$ sejam afetadas por anormalidades da função tireoidiana. Em um estudo brasileiro envolvendo 127 gestantes normais, o diagnóstico de hipotireoidismo foi realizado em três delas $(2,4 \%)^{6}$.

Embora existam algumas evidências de que discretas alterações tireoidianas predisponham à depressão, a associação entre anormalidades específicas envolvendo a tireoide e a ocorrência de quadros depressivos permanece pouco compreendida. As alterações comportamentais vêm sendo associadas ao hipotireoidismo há mais de $50 \mathrm{anos}^{7}$, independentemente da gravidade da doença, quer no mixedema, quer no hipotireoidismo subclínico. Uma pesquisa realizada no Brasil $^{4}$ destacou que o hipotireoidismo esteve significativamente associado à depressão. Em uma revisão ${ }^{8}$, a prevalência de sintomas depressivos entre casos de hipotireoidismo foi de aproximadamente $50 \%$, sendo $40 \%$ destes sujeitos considerados acometidos de depressão.

Dois estudos demonstraram maior prevalência de depressão e ansiedade em grupos de pacientes com hipotireoidismo subclínico quando comparados a controles eutireoidianos, indicando a necessidade de ensaios clínicos para avaliar uma possível melhora com uso de levotiroxina ${ }^{9,10}$. Em um estudo clínico realizado na Inglaterra ${ }^{11}$, o número de pacientes hipotireoideos controlados com queixa de sintomas psiquiátricos foi $26 \%$ maior em comparação a pacientes eutireoidianos. Em pacientes psiquiátricos, a taxa de hipotireoidismo tem variado de 0,5 a $8 \%{ }^{12}$. Outro estudo $^{13}$ mostrou alternativas para melhorar sintomas psicológicos e somáticos associando liotironina (T3) à levotiroxina (T4) em pacientes hipotireoideos.

Alguns pesquisadores tentam potencializar o tratamento antidepressivo em pacientes com sintomas refratários utilizando levotiroxina em doses suprafisiológicas, mesmo na ausência de doença tiroidiana ${ }^{14,15}$. Também foi demonstrado, pela tomografia por emissão de pósitrons (PET) com 18-fluoro-deoxi-glicose, que em pacientes do sexo feminino eutireoidianas, com depressão bipolar houve melhora global da atividade neurológica funcional após início da terapia de reposição hormonal com levotiroxina sintética em doses variadas.

Este estudo teve por objetivo avaliar a associação entre o hipotireoidismo e a ocorrência de sintomas depressivos e ansiosos em pacientes ambulatoriais.

\section{Métodos}

Foi realizado um estudo do tipo caso-controle no Hospital Universitário Gaffrée e Guinle (HUGG), no município do Rio de Janeiro, no período de Julho de 2006 a Março de 2008.

Foram selecionadas, no ambulatório de endocrinologia, 50 pacientes para compor o grupo de casos. Foram utilizados os seguintes critérios de inclusão: sexo feminino; hipotireoidismo primário independentemente da etiologia; idade entre 18 e 65 anos; dosagem do TSH até oito semanas antes do dia da inclusão no estudo; uso regular de levotiroxina sódica, por um período mínimo de três meses, em uma das seguintes preparações comerciais: Syntroid ${ }^{\circledR}$, Puran T4 ${ }^{\circledR}$, Euthyrox ${ }^{\circledR}$. Os pacientes hipotireoideos responderam aos questionários após a análise do $\mathrm{TSH}$, realizado de rotina pela técnica de quimiluminescência, com valores de referência de 0,3 a 5,0 mcUI/mL para padrão de normalidade.

O Grupo Controle foi composto por 50 acompanhantes, visitantes, pacientes do ambulatório de clínica médica e funcionários do HUGG. Foram utilizados os seguintes critérios de inclusão: sexo feminino, idade entre 18 e 65 anos, ausência de história pessoal prévia de doença tireoidiana; dosagem de TSH com valores de 0,4 a $4,0 \mu \mathrm{UI} / \mathrm{mL}$. Os controles responderam aos questionários e realizaram coleta de sangue venoso periférico no mesmo dia. As amostras de sangue foram processadas após calibração para evitar qualquer erro pré-analítico. Foi utilizado o aparelho Imulite ${ }^{\circledR}$ que opera pela técnica de quimiluminescência de terceira geração, com valores de referência de 0,4 a $4,0 \mu \mathrm{UI} / \mathrm{mL}$ e variações intraensaio e interensaio, respectivamente, de 3,8 a $12,5 \%$ e 4,6 a $12,5 \%$.

Nos dois grupos foram aplicados os seguintes critérios de exclusão: indivíduos analfabetos, uso regular de medicação antidepressiva ou ansiolítica em um período de até seis meses anterior ao estudo, uso de qualquer outro tipo de medicamento com ação no sistema nervoso central, inclusive glicocorticoides, gravidez e alcoolismo.

Havendo concordância em participar do estudo, após assinado o termo de consentimento livre e esclarecido, foram realizadas entrevista e aplicação dos testes psicológicos.

Os instrumentos de avaliação dos sintomas depressivos e ansiosos foram, respectivamente, as Escalas de Depressão de Ansiedade de Beck ${ }^{16}$, ambos validados para a língua portuguesa. Os questionários foram autoaplicados. A fim de evitar distorções no protocolo, o pesquisador principal aplicou todos os questionários, tendo conhecimento quando se tratava de um caso ou um controle.

As características demográficas e epidemiológicas avaliadas foram: raça/cor (conforme padronizado pelo 
Tabela 1 - Características demográficas e epidemiológicas dos casos e controles

\begin{tabular}{|c|c|c|c|c|c|}
\hline \multirow{3}{*}{$\begin{array}{l}\text { Variável } \\
\text { Média de idade } \\
\end{array}$} & \multicolumn{2}{|c|}{ Casos $(n=50)$} & \multicolumn{2}{|c|}{ Controles $(n=50)$} & \multirow{3}{*}{$\begin{array}{c}\text { Valor de } \mathrm{p} \\
0,3\end{array}$} \\
\hline & \multicolumn{2}{|c|}{$47,7 \pm 10,3$} & \multicolumn{2}{|c|}{$45,6 \pm 10,0$} & \\
\hline & $n$ & $\%$ & $n$ & $\%$ & \\
\hline \multicolumn{6}{|l|}{ Raça/cor } \\
\hline Branca & 32 & 64 & 33 & 66 & \multirow{3}{*}{1,0} \\
\hline Parda & 12 & 24 & 11 & 22 & \\
\hline Negra & 6 & 12 & 6 & 12 & \\
\hline Solteira & 8 & 16,0 & 10 & 20 & \multirow{3}{*}{0,6} \\
\hline Divorciada/separada & 7 & 14 & 10 & 20 & \\
\hline Viúva & 3 & 6 & 1 & 2 & \\
\hline \multicolumn{6}{|l|}{ Escolaridade } \\
\hline $1^{\circ} \mathrm{grau}$ incompleto & 9 & 18 & 7 & 14 & 0,2 \\
\hline Sim & 4 & 8,0 & 10 & 20 & \multirow{2}{*}{0,07} \\
\hline Não & 46 & 92 & 40 & 80 & \\
\hline \multicolumn{6}{|l|}{ Trabalho } \\
\hline Sim & 31 & 62,0 & 37 & 74,0 & \multirow{2}{*}{0,1} \\
\hline Não & 19 & 38,0 & 13 & 26,0 & \\
\hline IMC médio & \multicolumn{2}{|c|}{$27,5 \pm 5,7$} & \multicolumn{2}{|c|}{$26,8 \pm 5,6$} & 0,5 \\
\hline \multicolumn{6}{|l|}{ Obesidade } \\
\hline Sim & 32 & 64,0 & 30 & 60,0 & \multirow{2}{*}{0,4} \\
\hline Não & 18 & 36,0 & 20 & 40,0 & \\
\hline \multicolumn{6}{|l|}{ Menopausa } \\
\hline
\end{tabular}

IBGE); idade; menopausa; índice de massa corporal (IMC); etilismo (ingestão de uma ou mais doses de qualquer bebida alcoólica por dia); estado civil e situação profissional. Para a escala de ansiedade de Beck, foram considerados ansiosos os pacientes que apresentaram pontuação maior ou igual a 20 e normais aqueles com pontuação menor ou igual a $19^{17}$. Já para a escala de depressão de Beck, pacientes com pontuação até 10 foram considerados normais, aqueles com valores entre 11 e 20 foram classificados como possuidores de leve transtorno de humor ou distímicos e os com valores maiores que 20, como prováveis pacientes depressivos $^{18}$.

Ao delinear o estudo, estimou-se que, para detectar uma prevalência de depressão de $50 \%^{8}$ nas pacientes com hipotireoidismo, e $16^{5}$ a $18 \%{ }^{4}$ entre as mulheres com função tiroidiana normal, considerando-se um erro alfa de 0,05 e um erro beta de $10 \%$, seria necessária a inclusão de 50 casos e 50 controles.

Para a análise estatística, foi utilizado o programa SPSS, versão 14. Foram calculadas as prevalências relativas de depressão e de ansiedade por meio da razão das prevalências dessas condições entre casos e controles. As comparações entre variáveis contínuas foram realizadas por meio do teste de Mann-Whitney. Para comparação de proporções entre dois grupos, foram utilizados os testes $\chi^{2}$ ou exato de Fisher, conforme indicação. Os valores de $\mathrm{p}<0,05$ foram associados a diferenças consideradas significativas.

O estudo foi aprovado pela Comissão de Ética em Pesquisa do Hospital Universitário Gaffrée e Guinle, sob número 0060.0.328.000-07, tendo sido respeitadas as recomendações do Conselho Nacional de Ética em Pesquisa (CONEP).

\section{Resultados}

Os dados demográficos e epidemiológicos dos casos e controles encontram-se resumidos na Tabela 1. Não foram verificadas diferenças significativas nos itens analisados, sugerindo tratar-se de grupos semelhantes com relação a esses aspectos.

$\mathrm{Na}$ comparação entre os dois grupos quanto aos sintomas depressivos (Tabela 2), os pacientes foram classificados de duas formas, conforme os valores das variáveis numéricas obtidas, em normais, distímicos ou depressivos e com depressão e sem depressão. Não houve diferença 
Tabela 2 - Prevalência de sintomas depressivos e ansiosos em casos e controles

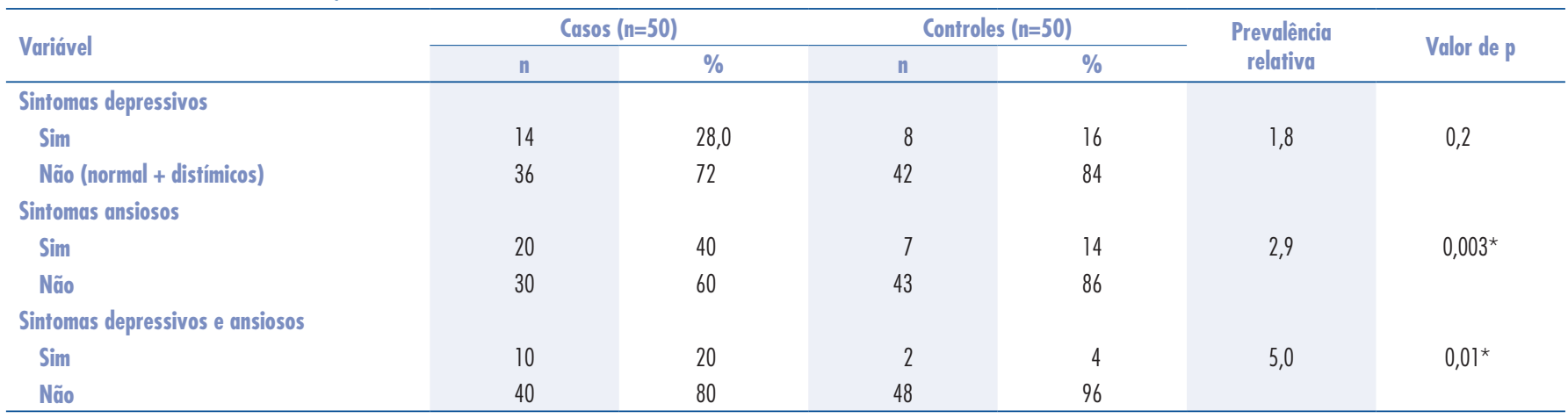

*Diferença significativa.

significativa na intensidade dos sintomas depressivos quando foram comparados os valores obtidos para os casos e os controles $(p=0,1)$. Vale ressaltar que, apesar de não haver diferença significativa entre hipotireoideos e eutireoidianos quanto aos sintomas depressivos, a prevalência relativa foi de 1,75 , indicando uma chance de apresentar depressão $75 \%$ maior nos pacientes hipotireoideos.

Com relação aos sintomas ansiosos, observou-se que $40 \%$ dos casos apresentaram escores compatíveis com ansiedade clínica na Escala de Beck contra 14\% dos controles ( $\mathrm{p}=0,003)$. Além disso, a prevalência relativa para sintomas ansiosos foi de 2,9, indicando um aumento de quase três vezes na probabilidade de se observar ansiedade clínica nos pacientes hipotireoideos quando comparados aos eutireoidianos.

Quando analisada a prevalência de pacientes com concomitância de sintomas de ansiedade e depressão (valores simultaneamente superiores aos pontos de corte das duas escalas), foram encontrados 12 casos, sendo 10 no grupo de hipotireoideos e 2 no grupo de controles. Esse fato indica uma probabilidade cinco vezes maior (prevalência relativa $=5$ ) de se observarem sintomas ansiosos e depressivos no grupo de hipotireoideos do que no Grupo Controle ( $\mathrm{p}=0,01)$.

Os níveis séricos de TSH mostraram variação de 0,58 a $3,86 \mathrm{mcU} / \mathrm{mL}$, com mediana de $1,7 \mathrm{mcU} / \mathrm{mL}$, nos eutireoidianos e de 0,35 e $6,91 \mathrm{mcU} / \mathrm{mL}$, com mediana de 1,81 $\mathrm{mcU} / \mathrm{mL}$, nos pacientes hipotireoideos. Entre os pacientes hipotireoideos, após estratificação conforme os níveis de TSH ( $\leq 5$ versus $>5 \mathrm{mcU} / \mathrm{mL}$ ), observou-se não haver diferença significativa na frequência dos sintomas depressivos $(16,7$ versus $29,5 \%$, respectivamente; $p=0,4)$ ou ansiosos (38,6 versus 50,0\%, respectivamente; $p=0,59)$.

\section{Discussão}

Os estudos que avaliaram a presença de alterações do humor em pacientes com hipotireoidismo apresentaram resultados contraditórios, pois utilizaram critérios metodológicos muito distintos, não sendo possível, portanto, compará-los. Provavelmente esse fato decorre da diversidade das características clínicas dos pacientes hipotireoideos, da ausência de estudos comparando casos e controles e da grande quantidade de escalas disponíveis para quantificar sintomas depressivos e ansiosos, muitas delas não traduzidas ou validadas para a língua portuguesa.

No presente estudo, foi observada uma prevalência $75 \%$ maior de sintomas depressivos entre os casos do que entre os controles, embora sem significância estatística na comparação entre os grupos, enquanto a prevalência de ansiedade foi quase três vezes maior entre os casos, com diferença significativa. Placidi et al. ${ }^{19}$ também demonstraram maior prevalência de transtornos psiquiátricos em pacientes hipotireoideos quando comparados a controles, porém nesse estudo foi utilizada uma entrevista estruturada do Manual Estatístico de Transtornos Mentais.

Em outro estudo, foram observados escores elevados de pontuação na Escala de Ansiedade de Beck em pacientes com hipotireoidismo subclínico sem uso de medicamentos quando comparados a pacientes eutireoidianos ${ }^{9}$, e a alta prevalência de sintomas ansiosos pode levar a uma piora na qualidade de vida, apontando a necessidade de tratamento nesses pacientes.

Neste estudo, foram dosados os níveis de TSH isoladamente como método para avaliação de hipotireoidismo primário. Uma vez que a secreção de $\mathrm{T}_{3} \mathrm{e} \mathrm{T}_{4}$ pela glândula tireoide é regulada pela secreção hipofisária de TSH de forma log-linear ${ }^{20}$, quaisquer alterações nos níveis de hormônios tireoidianos podem levar a grandes alterações nas concentrações séricas de TSH, fazendo do TSH o melhor método para se quantificarem alterações discretas da função tireoidiana. Observou-se que a mediana do TSH foi de $1,8 \mathrm{mcU} / \mathrm{mL}$ nos pacientes hipotireoideos e de $1,7 \mathrm{mcU} /$ $\mathrm{mL}$ nos pacientes eutireoidianos, resultados compatíveis com outro estudo recentemente publicado ${ }^{21}$.

Não foi possível confirmar a hipótese de que o valor de TSH atue como variável influenciadora ou determinante de aumento de sintomas depressivos e ansiosos. Em um estudo multicêntrico envolvendo 597 pacientes hipotireoideos e 551 pacientes eutireoidianos, foi verificado que, independentemente 
do valor de TSH, os pacientes hipotireoideos apresentaram um maior prejuízo do bem-estar psicológico quando pareados com controles do mesmo sexo e faixa etária, sem considerar outros fatores socioculturais ${ }^{11}$. Entre pacientes hipotireoideos com conhecimento de sua condição clínica, também se observou risco aumentado para depressão ou ansiedade independentemente dos valores de $\mathrm{TSH}^{22}$. Porém, quando somente os casos de hipotireoidismo subclínico sem uso de medicação, e portanto com TSH alterado, foram comparados com pacientes eutireoidianos, não foi encontrada diferença significativa quanto à presença de alterações depressivas e ansiosas. Nesse mesmo estudo ${ }^{22}$, foi observado ainda que a concomitância de sintomas depressivos e ansiosos torna quase impossível afirmar se um paciente é puramente depressivo ou ansioso. Isso acontece porque os dois construtos (depressão e ansiedade) podem ser componentes de um só processo psicológico. Na prática clínica, depressão e ansiedade se apresentam na maior parte das vezes de forma indissociável. Isso pode explicar em parte o fato de não ter sido encontrada diferença significativa na prevalência de depressão no grupo de hipotireoideos em relação aos eutireoidianos no atual estudo. Porém, quando foi analisada a frequência de sintomas depressivos e ansiosos simultaneamente (pontuação elevada nas duas escalas), observou-se que sua probabilidade é cinco vezes maior no grupo dos pacientes hipotireoideos do que entre controles.

É importante destacar que, embora não tenha sido observada uma diferença significativa na prevalência de depressão entre casos e controles, estima-se que sua prevalência na população geral varie entre 3 e $12 \%{ }^{23}$. A prevalência de depressão encontrada nos pacientes hipotireoideos deste estudo (28\%) foi maior do que o dobro do valor máximo esperado na população geral (12\%). Entretanto, diferentemente do esperado, a prevalência de depressão entre os controles (16\%) foi também ligeiramente superior à da população geral, o que pode ter enviesado os resultados do presente estudo para a não-comprovação da principal hipótese formulada, que era haver associação entre depressão e hipotireoidismo.

É importante destacar que, entre os controles, um percentual elevado das mulheres encontrava-se na peri ou pós-menopausa (média de idade $45,6 \pm 10$ anos), quando a depressão é mais frequente, o que também pode ter reduzido a capacidade deste estudo de diferenciar os casos dos controles no que diz respeito à prevalência de depressão. Dados já publicados apontam o fato que de $36,8^{24} \mathrm{a}$ $41,8 \%{ }^{25}$ das mulheres nesse estado menopausal podem apresentar sintomas de depressão. Além disso, estima-se que a prevalência de desordens depressivas entre pacientes consultando um ginecologista pode variar de 10 a $40 \%$. Entre mulheres mexicanas que frequentavam hospitais públicos, observou-se que 32,6\% eram depressivas ${ }^{26}$. Já em estudo de base populacional com mulheres brasileiras de meia idade, a prevalência de sintomas depressivos foi de $45,7 \%{ }^{2}$. Deve-se salientar que esses estudos utilizaram diferentes metodologias para avaliação da depressão.

Por fim, a inclusão de funcionárias do Hospital no Grupo Controle pode ter inserido um viés de seleção no presente estudo, uma vez que profissionais de saúde podem apresentar sintomas depressivos e ansiosos mais frequentemente do que a população geral, o que representa uma limitação para os resultados descritos. Deve-se levar em consideração o tamanho amostral do presente estudo. Estudos futuros incluindo um número maior de observações poderão estabelecer melhor a relação entre hipotireoidismo e sintomas depressivos e ansiosos.

Conclui-se que a probabilidade de mulheres com hipotireoidismo apresentarem concomitantemente sintomas ansiosos e depressivos é cinco vezes maior do que em mulheres eutireoidianas. Quando analisados individualmente, os sintomas ansiosos foram três vezes mais frequentes entre os casos do que entre os controles, enquanto os sintomas depressivos mostraram frequência $75 \%$ mais elevada entre as pacientes com hipotireoidismo. Devido às altas prevalências de hipotireoidismo e depressão observadas na prática clínica, sintomas depressivos devem ser investigados em pacientes com disfunção tireoidiana, e pacientes deprimidos devem ser testados com TSH.

\section{Agradecimentos}

Ao doutor Fernando C. M. Ferreira por, gentilmente, ceder o material e pessoal necessários para os testes de TSH realizados nas pacientes.

\section{Referências}

1. Redmond GP. Hypothyroidism and women's health. Int J Fertil Womens Med. 2002;47(3):123-7.

2. Guimarães JM, de Souza Lopes C, Baima J, Sichieri R. Depression symptoms and hypothyroidism in a population-based study of middle-aged Brazilian women. J Affect Disord. 2009;117/12): 120-3.
3. Tavares AB. Impacto do hipotireoidismo entre mulheres climatéricas. Rev Bras Ginecol Obstet. 1999;21 (9):560.

4. Franco VA. Prevalência de hipotireoidismo e características clínicas associadas à hipofusão tireoideana entre mulheres climatéricas: um estudo de base hospitalar. Rev Bras Ginecol Obstet. $2004 ; 26(3): 255$. 
5. Nahas EAP, Nahas-Neto J, Santos PEMF, Mazeto GMFS, Dalben I, Pontes $A$, et al. Prevalência do hipotiroidismo subclínico e repercussões sobre o perfil lipídico e massa óssea em mulheres na pós-menopausa. Rev Bras Ginecol Obstet. 2005;27(8):467-72.

6. Rodrigues LP, Jorge SRPF, Roveran V, Yamano LM, Vásquez ML, Aoki T, et al. Quantificação de anticorpos antitireoperoxidase e antitireoglobulina, tireotrofina e tiroxina livre em gestantes normais. Rev Bras Ginecol Obstet. 2007;29(9):478-83

7. Teng CT, Humes EC, Demetrio FN. Depressão e comorbidades clínicas. Rev Psiquiatr Clín (São Paulo). 2005;32(3):149-59.

8. Boswell EB, Anfinson TH, Nemeroff CB. Depression associated with endocrine disorders. In: Robertson MM, Katona CLE, editors. Depression and physical illness. Chichester: Wiley; 1997. p. 256-92.

9. Sait Gonen M, Kisakol G, Savas Cilli A, Dikbas O, Gungor K, Inal $A$, et al. Assessment of anxiety in subclinical thyroid disorders. Endocr J. 2004;51(3):311-5.

10. Bono G, Fancellu R, Blandini F, Santoro G, Mauri M. Cognitive and affective status in mild hypothyroidism and interactions with L-thyroxine treatment. Acta Neurol Scand. 2004;110(1):5966.

11. Saravanan P, Chau WF, Roberts N, Vedhara K, Greenwood R, Dayan CM. Psychological well-being in patients on 'adequate' doses of l-thyroxine: results of a large, controlled community-based questionnaire study. Clin Endocrinol (Oxf). 2002;57(5):577. 85.

12. Targum SD, Greenberg RD, Harmon RL, Kessler K, Salerian A, Fram $\mathrm{DH}$. Thyroid hormone and the TRH stimulation test in refractory depression. J Clin Psychiatry. 1984;45(8):345-6.

13. Escobar-Morreale HF, Botella-Carretero Jl, Gómez-Bueno M, Galán JM, Barrios V, Sancho J. Thyroid hormone replacement therapy in primary hypothyroidism: a randomized trial comparing L-thyroxine plus liothyronine with L-thyroxine alone. Ann Intern Med. 2005;142(6):412-24.

14. Baver $M$, Berghöfer A, Bschor T, Baumgartner A, Kiesslinger U, Hellweg R, et al. Supraphysiological doses of L-thyroxine in the maintenance treatment of prophylaxis-resistant affective disorders. Neuropsychopharmacology. 2002;27(4):620-8.
15. Łojko D, Rybakowski JK. L-thyroxine augmentation of serotonergic antidepressants in female patients with refractory depression. J Affect Disord. 2007;103(1-3):253-6.

16. Beck TA, Rush AJ, Shaw BF, Emery G. Terapia cognitiva da depressão. Porto Alegre: Artmed; 1997.

17. Andrade LHSG, Gorenstein C. Aspectos gerais das escalas de avaliação de ansiedade. Rev Psiquiatr Clín (São Paulo). $1998 ; 25(6): 285-90$

18. Gorenstein C, Andrade L. Validation of a Portuguese version of the Beck Depression Inventory and the State-Trait Anxiety Inventory in Brazilian subjects. Braz J Med Biol Res. 1996;29(4):453-7.

19. Placidi GP, Boldrini M, Patronelli A, Fiore E, Chiovato L, Perugi $C$, et al. Prevalence of psychiatric disorders in thyroid diseased patients. Neuropsychobiology. 1998;38(4):222-5.

20. Nutt DJ, Bell CJ, Malizia AL. Brain mechanisms of social anxiety disorder. J Clin Psychiatry. 1998;59 Suppl 17:4-1 1.

21. Sichieri R, Baima J, Marante T, de Vasconcellos MT, Moura AS, Vaisman M. Low prevalence of hypothyroidism among black and Mulatto people in a population-based study of Brazilian women. Clin Endocrinol (Oxf). 2007;66(6):803-7.

22. Engum A, Bjoro T, Mykletun A, Dahl AA. An association between depression, anxiety and thyroid function - a clinical fact or artefact? Acta Psychiatr Scand. 2002;106(1):27-34.

23. Dratcu L, da Costa Ribeiro L, Calil HM. Depression assessment in Brazil. The first application of the Montgomery-Åsberg Depression Rating Scale. Br J Psychiatry. 1987;150:797-800.

24. Polisseni AF, Araújo DAC, Polisseni F, Mourão Junior CA, Polisseni J, Fernandes ES, et al. Depressão e ansiedade em mulheres climatéricas: fatores associados. Rev Bras Ginecol Obstet. 2009:31(1):28-34.

25. Timur S, Sahin NH. The prevalence of depression symptoms and influencing factors among perimenopausal and postmenopausal women. Menopause. 2010;17(3):545-51.

26. Alvarado-Esquivel C, Sifuentes-Alvarez A, Estrada-Martínez $S$, Salas-Martínez C, Hernández-Alvarado AB, Ortiz-Rocha SG, et al. Prevalence of postnatal depression in women attending public hospitals in Durango, Mexico. Gac Med Mex. 2010;146(1):1-9. 